\title{
"Three Preventions" System Design Based On Single-Chip Microcomputer (SCM) Control
}

\author{
Jiaxiong $\mathrm{Wen}^{1, \text { a }}$ \\ ${ }^{1}$ Sichuan Information Technology College, China \\ a472108923@qq.com
}

Keywords: SCM; Sensor; "Three Preventions" System.

\begin{abstract}
With the rapid development of social economy, Science and technology level are increasingly improving, especially accelerated pace of life, People demand for fireproof, anti-theft and anti-gas equipment is greatly increased, and for theft, fire and other accident monitoring and alarm system requirements are also higher. In the research of this thesis is based on AT89S52 SCM as the core of the fireproof and anti-theft alarm system, not only can call the police, but also can display the alarm information category. This system selects the smoke sensor, gas sensor, pyroelectric infrared sensor for detecting element, and uses multi-sensor information fusion technology designed to be used for fire and theft alarm in small-scale agricultural production, etc. This design is reasonable, the product performance and reliable, and can replace some expensive product.
\end{abstract}

\section{The basic principle of "three prevention" system design}

This design includes two parts of hardware and software design. Module is divided into smoke acquisition, temperature acquisition, $\mathrm{AD}$ conversion, sound and light alarm module sub functions.

The infra-red anti-theft alarm system consists of pyroelectric infrared sensor, alarm, SCM control circuit, LED control circuit and related control management software. User terminals complete information acquisition, processing, data transmission, function setting, local alarm, etc.

Functions of the system implementation. When the detector working, pyroelectric infrared sensor to detect movement if someone broke into, infrared sensor set on monitoring points will transform IR radiation of the human body into electrical signals, electrical signal via an amplifier circuit, comparator circuit to the threshold switch, open the gate valve send TTL level to AT89S52 SCM, after a single-chip processing operations, drive to perform alarm circuit warning voice. If detected by temperature sensor DS18B20 collecting temperature above or below the alarm value, the acousto-optic alarm. Also with MQ-2 smoke sensor detects smoke, after ADC0832 conversion to the microcontroller, and then to drive the acousto-optic alarm. With MQ - 5 combustible gas sensor detecting combustible gases such as methane, when the gas leak, sound and light alarm driving.

Infrared is concealment, design a beam of infrared light in the open air protection place, it can be easily detected if anyone out. Design key points of such kind of device: one is to effectively judge whether there is someone entering and the other is as far as possible to increase the protection scope. Of course, the stability and reliability of the system is also an important indicator.

\section{Hardware structure design of "three prevention" system}

This system has the function of anti-theft and anti-fire and prevents the poisonous gases such as carbon monoxide, Smoke sensors and combustible gas sensors can detect smoke and carbon monoxide concentration. If the concentration of carbon monoxide exceeded, the microcontroller will drive the acousto-optic alarm, when the pyroelectric infrared sensor detects someone or when the room there is a fire phenomenon, SCM pin level conversion which also drives the acousto-optic alarm circuit alarming. The system hardware structure diagram is shown in FIG 1. 


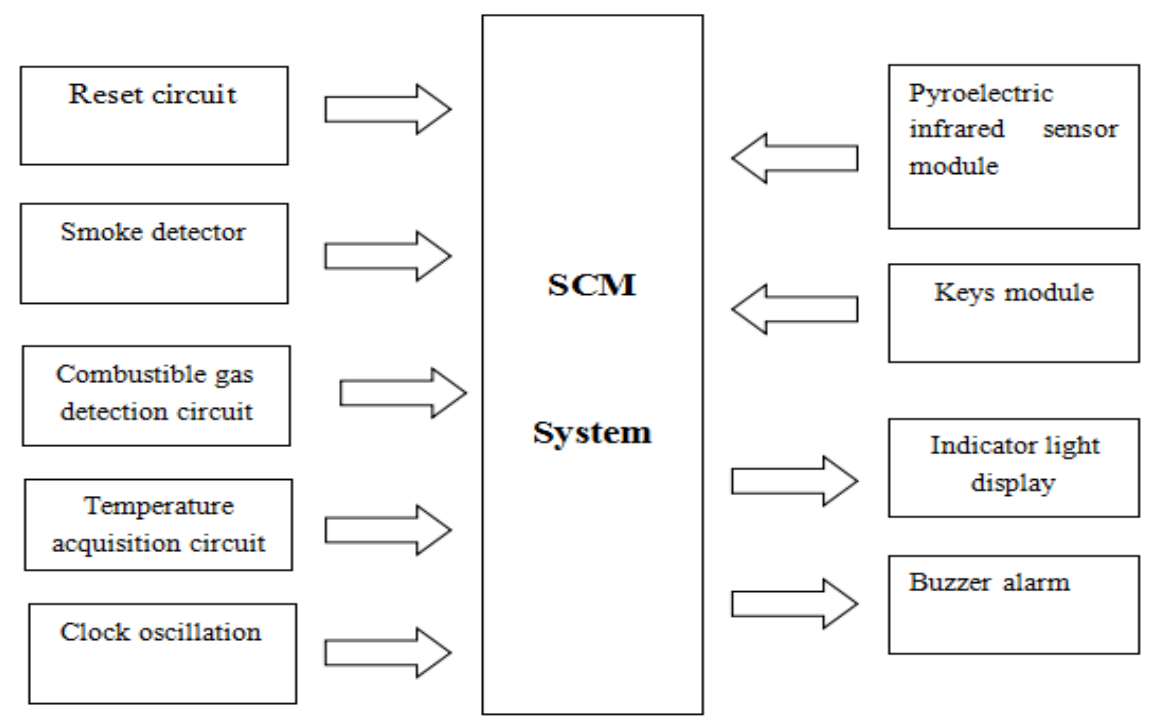

Fig. 1 system hardware structure diagram

\section{"Three prevention" system software design}

The main program flow chart is shown in FIG 2. The system enters monitoring status after the program initialization.

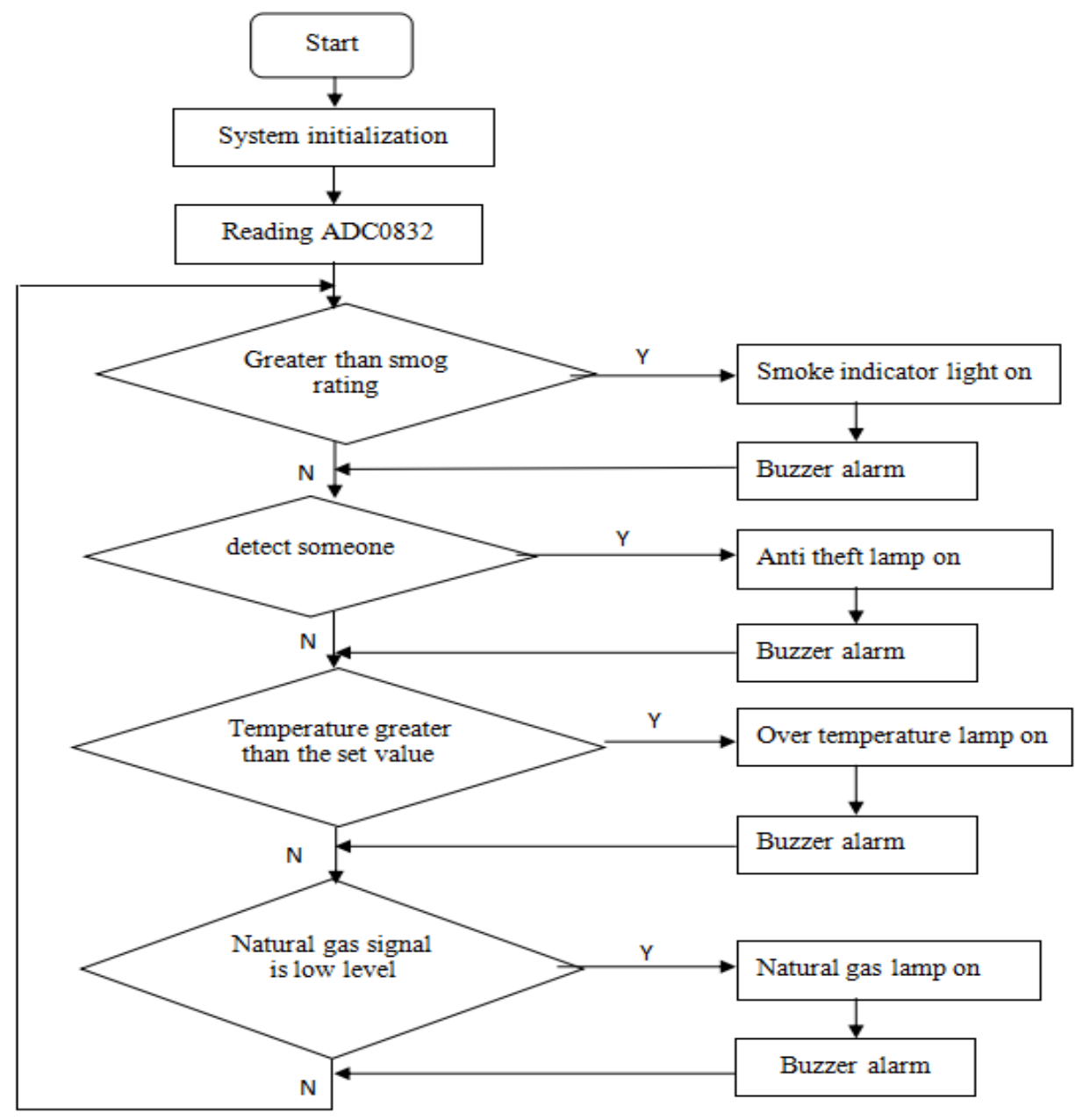

FIG 2 The main program flow chart

The main function of the temperature subroutine is to read 9 gigabytes of RAM, when reading out it required CRC checking and no temperature data rewriting if checksum error. For each command of DS18B20 timing requirements are particularly stringent, it must in accordance with the required 
sequence to achieve the desired purpose. Meanwhile, We should pay attention to read incoming data is High digit behind low digit, a total of 12 digits, four decimal and integer seven, and a sign bit.

Smoke A / D conversion flow chart shown in FIG 3. The main component of smog acquisition circuit is smoke sensor and smoke signals collected by the smoke sensor, and then convert into analog electrical signals. A/D converter circuit convert this analog electrical signal to digital signal which SCM can be identified for data analysis to determine whether greater than or equal to a preset value, if greater than or equal to the buzzer alarm.

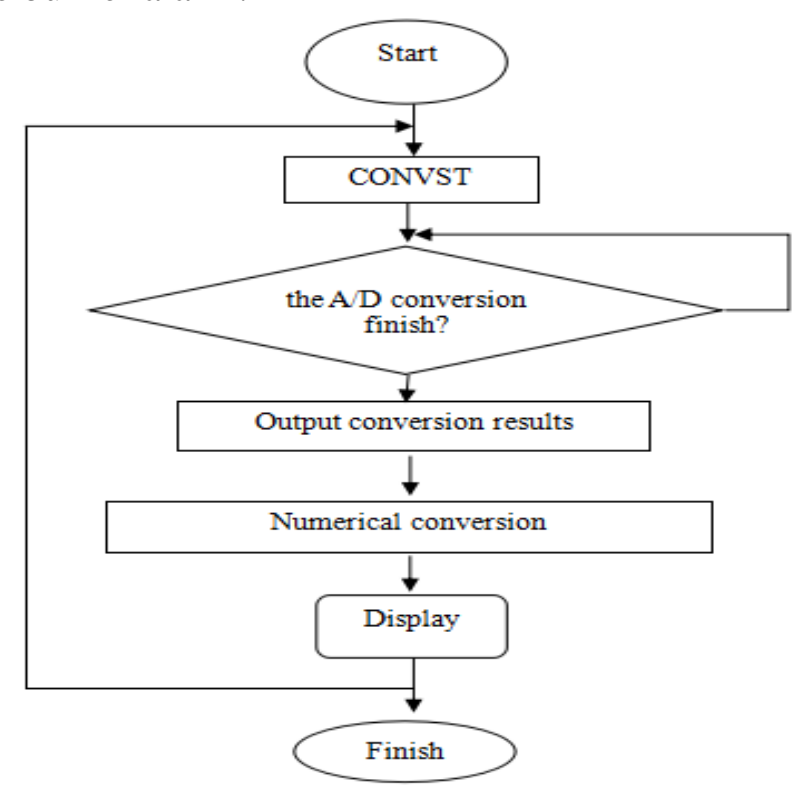

FIG 3 Smoke A / D conversion flow chart

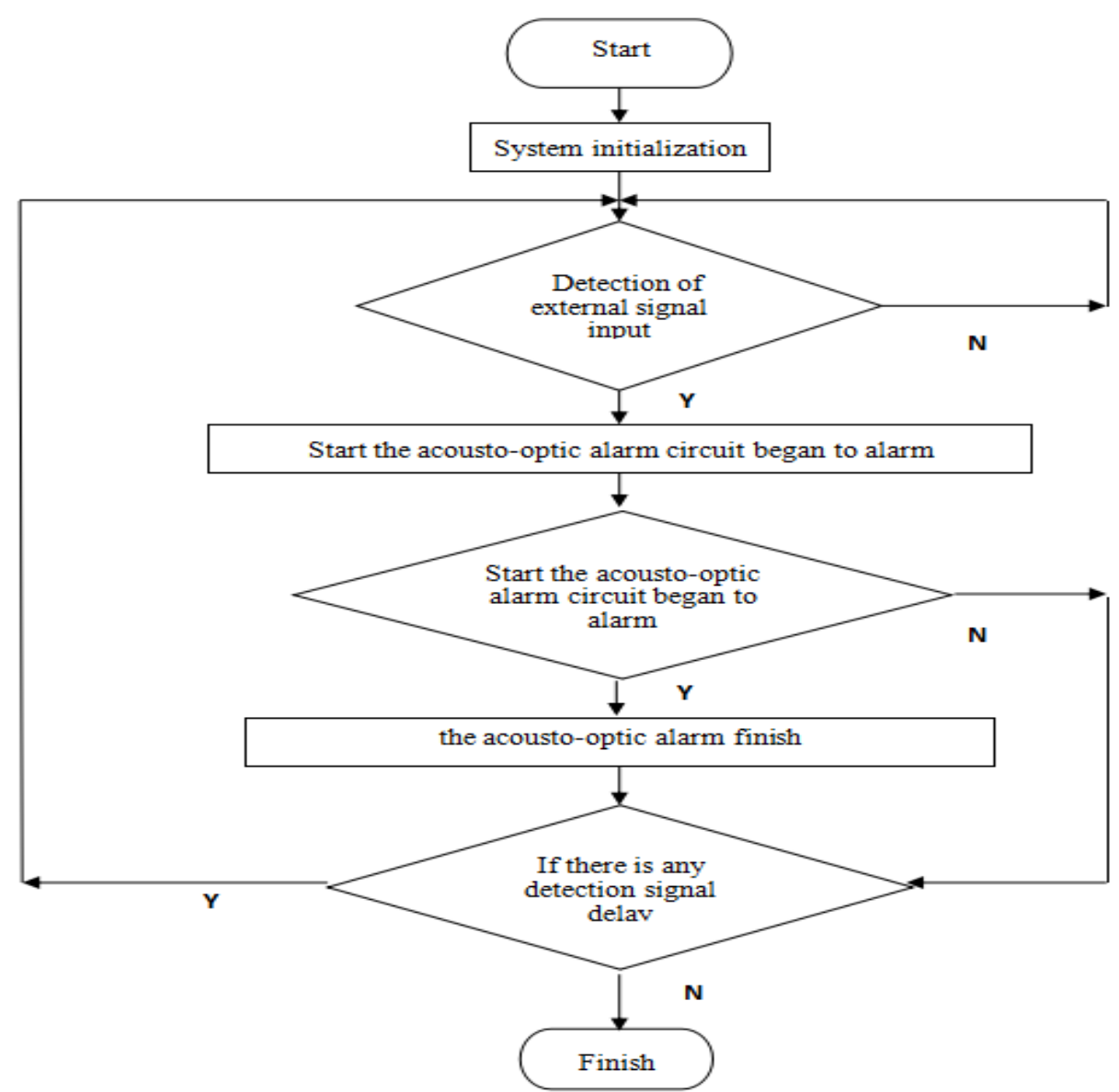

FIG 4 Pyroelectric module work flow chart 
Pyroelectric module work flow chart shown in FIG 4, the whole system is working in the system software control. Infrared sensor set on monitoring points will transform IR radiation of the human body into electrical signals, electrical signal via an amplifier circuit and send TTL level to AT89S51 SCM, Drive circuit control signal is amplified and promote the acousto-optic alarm. The alarm detect whether there is any person after a period of time, if nobody is automatically lifted and continues to monitor waiting for the next alarm. When the alarm eliminate, reset circuit to reset the system.

\section{Conclusion}

The design is study for a kind of wireless intelligent anti-fire and anti-theft alarm system based on SCM technology. The alarm work by AT89S52 SCM as the core processor, an external pyroelectric infrared sensor, smoke sensor, combustible gas sensor and temperature sensor, the alarm can detect infrared radiation emitted by human body with non-contact way, and converted into a corresponding electrical signal output. It also can effectively suppress infrared light and visible light interference which over the personal radiation wavelength. This alarm could judge the level and set the indoor temperature according to smog situation and also detect toxic gas and then alarming. The biggest characteristic of this alarm is that users can easily understand and operate it flexible. And easy installation, high intelligence, low rate of false positives. With the rapid development of science and technology, and the enhancement of the modern people security awareness, we believe that the alarm will get deeper applications in a wide area.

\section{References}

[1] Chaoqing Li. Microcontroller principles and interface technology (The concise revision edition) Hangzhou: Beijing University of Aeronautics and Astronautics Press, 1998

[2] Guangdi Li. SCM Basic. [M].Beijing: Beijing University of Aeronautics and Astronautics Press

[3] Shi Yan. Fundamentals of Digital electronic techniques. (third edition). Beijing: Higher Education Press, 1989

[4] Donghai Shi, etc. The Data Communication Technology of Single-chip Processor for Both Beginners \& Experts, Xian: Xian University of electronic science and technology press, 2002

[5] Zhongfei Wang, Fang Xu. MCS - 51 SCM principle and embedded systems applications. [M]. Xian: Xian University of electronic science and technology press, 2007

[6] Chaoyang Cai. SCM control and practice project [M]. Beijing: Beijing University of Aeronautics and Astronautics Press, 2006

[7] Yi Gang, Xiyuan Peng, Xiaojun Tan, etc. MSC-51 SCM application design [M]. Harbin: Harbin Institute of Technology Press, 1999

[8] Jian Zhou. SCM C language easy start. [M]. Beijing: Beijing University of Aeronautics and Astronautics Press , 2006

[9] Quanli Li, Rongqiang Chi. SCM Theory and Interface Technology [M]. Beijing: Higher Education Press, 2004

[10] Lifeng Mei, Yanqiu Wang. SCM Theory and Interface Technology (revision). [M]. Beijing: Tsinghua University Press; Beijing jiaotong university press, 2006

[11] Shi Yan. Fundamental Digital Electronic Technique. [M]. Beijing: Higher Education Press, 1998

[12] Changchu Liao. PRIFOIBUS [J]. Electrotechnics, 1999 
[13] Yihuai Wang, Xiaoshseng Liu. Embedded application technology Primer. Beijing: Tsinghua University Press, 2005

[14] Beibei, etc. SCM knowledge and practice. Beijing: Beijing University of Aeronautics and Astronautics Press, 2006. 